\title{
Ascídias (Tunicata, Ascidiacea) introduzidas no Arquipélago de Alcatrazes, São Paulo
}

\author{
Rosana M. da Rocha \& Nadia Y. K. Bonnet
}

Laboratório de Ecologia e Sistemática de Ascidiacea, Departamento de Zoologia, Universidade Federal do Paraná, Caixa Postal 19020, 81531-980 Curitiba, PR, Brasil. (rmrocha@ufpr.br; nyk_bonnet@yahoo.com.br)

\begin{abstract}
Introduced ascidians (Tunicata, Ascidiacea) in the Arquipélago de Alcatrazes, State of São Paulo, Brazil. Arquipélago de Alcatrazes $\left(24^{\circ} 06^{\prime} \mathrm{S}, 45^{\circ} 42^{\prime} \mathrm{W}\right)$ is $36 \mathrm{~km}$ off the coast and it is an Ecological Station since 1987. It is also located near the port region of São Sebastião, São Paulo, hence subjected to introduction of exotic species. Forty samples were taken in the principal island of the archipelago to detect possible introductions. Fifteen samples were from Baía do Oratório and 25 from Saco do Funil. Twenty four species distributed in seven families were found. Only five of them were classified as native, four are Atlantic but cryptogenic in the region because of the disjunct distribution; five are cryptogenic with world wide distribution; and one is a certain introduction, Ciona intestinalis (Linnaeus, 1767). There are other nine species that were not identified, a few of them possibly new species. The presence of one introduced species and the high number of cryptogenic species with strong evidence of introduction indicate that the islands are threatened by non-native fauna. The conservation of the Archipelago demands consideration of the port as one source of stress and the control of exotic species as an important aspect of the management procedures.
\end{abstract}

KEYWORDS. Urochordata, taxonomy, bioinvason, geographical distribution, Ciona intestinalis.

RESUMO. O Arquipélago de Alcatrazes ( $24^{\circ} 06^{\prime} \mathrm{S}, 45^{\circ} 42^{\prime} \mathrm{W}$ ) localiza-se a $36 \mathrm{~km}$ da costa e constitui uma Estação Ecológica desde 1987. Estando próximo à região portuária de São Sebastião, São Paulo, está sujeito à introdução de espécies exóticas. Com o objetivo de detectar se está ocorrendo ou não introdução de espécies, foram coletados 40 exemplares de ascídias da principal ilha do arquipélago, a Ilha de Alcatrazes, sendo 15 amostras da Baía do Oratório e 25 do Saco do Funil. Foram encontradas 24 espécies em sete famílias. Dentre estas, apenas cinco espécies podem ser consideradas como nativas para o Atlântico; quatro são atlânticas, mas criptogênicas na região devido à distribuição disjunta; cinco são classificadas como criptogênicas de ampla distribuição mundial; e uma pode ser considerada como um caso certo de introdução, Ciona intestinalis (Linnaeus, 1767). Há ainda nove espécies que não puderam ser identificadas, podendo algumas se tratar de espécies novas. A presença de uma espécie exótica e a grande quantidade de espécies criptogênicas com forte evidência de introdução são indícios de que as ilhas estão sendo ameaçadas por uma fauna não nativa. A conservação do Arquipélago deve levar em consideração a presença do porto como fonte de estresse e o controle das espécies introduzidas como parte do plano de manejo da Unidade de Conservação.

PALAVRAS-CHAVE. Urochordata, taxonomia, bioinvasão, distribuição geográfica, Ciona intestinalis.

O Arquipélago de Alcatrazes ( $24^{\circ} 10^{\prime}$ S , 45ำ $\left.70^{\prime} \mathrm{W}\right)$ encontra-se localizado na região norte do litoral paulista, a 36 quilômetros da costa, município de São Sebastião. É formado por cinco ilhas: Ilha da Sapata, do Paredão, do Porto, do Sul, e a principal, a Ilha de Alcatrazes. Além disso, o Arquipélago conta ainda com quatro ilhotas, cinco lajes e dois parcéis (Projeto Alcatrazes, 2008). Apesar do arquipélago constituir uma estação ecológica desde 1987, a Marinha possui alvos pintados nas rochas para exercícios de tiro desde o início dessa mesma década na região do Saco do Funil (Ilha de Alcatrazes). Esta prática acaba colocando em risco a flora e fauna locais, motivo pelo qual ambientalistas vêm tentando transformar o Arquipélago em Parque Nacional.

A diversidade marinha do local também sofre outro tipo de risco, a proximidade com o porto de São Sebastião $(45 \mathrm{~km})$. Portos são considerados um dos principais locais de entrada de espécies em novos ambientes (CARLTON \& Geller, 1993; Ruiz et al., 2000; Wasson et al., 2001). O porto de São Sebastião movimenta cerca de 400 mil toneladas por ano, importando e exportando produtos de vários países diferentes (Secretaria De Estado dos TRANSPORTES, 2008), o que torna a área exposta a possíveis alterações em sua diversidade marinha pela introdução de novas espécies.
Assim, espécies introduzidas são uma ameaça à diversidade local (Carlton \& Geller, 1993; Cohen \& CARLton, 1998). Entre os diversos organismos marinhos passíveis de transporte e com capacidade de invasão estão dinoflagelados, clorófitas, rodófitas, poríferos, cnidários, briozoários, anelídeos, moluscos, crustáceos, equinodermos e ascídias (CARLton \& Geller, 1993). São diversas as ocorrências de transporte antropogênico e introdução de ascídias nos oceanos (e.g. DAVIS \& DAVIS, 2005; LAMBERT, 2001; LAMBERT, 2004; LAMBERT, 2007; Minchin et al., 2006; Zvyagintsev et al., 2003).

Para a costa brasileira, Bostrichobranchus digonas Abbott, 1951 foi detectada na Baía de Paranaguá, sendo uma espécie estuarina na Flórida (Rocha, 2002); Styela plicata (Lesueur, 1823) e Ascidia sydneiensis Stimpson, 1855 são indicadas como introduções na região sul e sudeste do país (Rocha \& KREMER, 2005) e RoCHA \& FARIA (2005) registraram Aplidium pentatrema (Monniot, 1972) para o litoral paranaense, esta espécie só havia sido registrada em Bermudas e Guadalupe. Assim, casos de introdução envolvendo ascídias se mostram bastante comuns.

Considerando que para o litoral do Estado de São Paulo há um amplo registro de espécies de Ascidiacea (VAn Name, 1945; BJornberg, 1956; Millar, 1958; 
Rodrigues, 1962, 1966, 1977; Rocha \& MonNiot, 1993, 1995; Rodrigues \& RoCHA, 1993; Rodrigues et al., 1998; DiAs \& RoDRIGUES, 2004) cujos levantamentos incluem a região de São Sebastião, é possível realizar um acompanhamento da área e avaliar a possível introdução de novas espécies. Assim, este trabalho teve por objetivo estudar a fauna de ascídias no Arquipélago de Alcatrazes que, apesar da proximidade com o Porto de São Sebastião, tem uma circulação de barcos de recreio nas ilhas limitada pela Marinha do Brasil. Desta forma, espera-se encontrar uma fauna exclusiva de espécies nativas.

\section{MATERIAL E MÉTODOS}

Foram coletadas 40 amostras de ascídias em dois locais distintos do arquipélago: 15 na Baía do Oratório (02/05/2002) e 25 no Saco do Funil (03/05/2002). As coletas consistiram de mergulhos autônomos em profundidades entre oito e vinte metros. Os exemplares foram retirados do substrato com o auxílio de uma faca e colocados em recipientes etiquetados junto com água do mar e cristais de mentol para anestesia. Posteriormente as amostras foram fixadas com formaldeído 4\%. Em laboratório, os animais foram corados com Hematoxilina de Harris, dissecados e identificados sob lupa.

Para a identificação de espécies da família Didemnidae é necessária a análise por microscopia eletrônica das espículas calcárias presentes na túnica das colônias. Para tanto, partes de cada colônia foram fervidas em água sanitária até que houvesse a total dissolução da matéria orgânica e restasse apenas o material calcário. As espículas foram então lavadas duas vezes com água e duas vezes com álcool $70 \%$, armazenadas em álcool $90 \%$, e analisadas em microscópio eletrônico Jeol JSM-6360LV.

A distribuição geográfica das espécies foi utilizada como critério para classificação das mesmas em nativas, criptogênicas ou introduzidas: as primeiras são aquelas naturais da região; as segundas são as que ou possuem uma ampla distribuição geográfica ou os organismos estão em áreas distantes entre si e não se sabe qual é a área original, não podendo ser classificadas nem como nativas e nem como introduzidas; e as introduzidas são as espécies nativas de uma região, mas que são encontradas em outro local mesmo sem haver possibilidade de dispersão natural (CARLTON, 1996; RuIZ et al., 2000). Outros critérios utilizados neste trabalho foram informações sobre substrato de fixação e hábitat (espécies introduzidas são geralmente encontradas em substratos artificiais, em portos e marinas) e registros históricos, quando disponíveis.

Todo o material identificado foi depositado na coleção de Ascidiacea do Departamento de Zoologia da Universidade Federal do Paraná.

\section{RESULTADOS E DISCUSSÃO}

Foram encontradas 24 espécies (Tab. I), sendo apenas três ascídias simples, pertencentes às famílias

Tabela I. Espécies de ascídias encontradas em duas localidades do Arquipélago de Alcatrazes, São Paulo, com a respectiva quantidade de exemplares ( $\mathrm{C}$, criptogênica; I, introduzida; $\mathrm{N}$, nativa).

\begin{tabular}{|c|c|c|c|}
\hline & Classificação & Baía Oratório & Saco do Funil \\
\hline \multicolumn{4}{|l|}{ PHLEBOBRANCHIA } \\
\hline \multicolumn{4}{|l|}{ CIONIDAE } \\
\hline Ciona intestinalis (Linnaeus, 1767) & I & & 1 \\
\hline \multicolumn{4}{|l|}{ PEROPHORIDAE } \\
\hline Perophora viridis Verril, 1871 & $\mathrm{~N}$ & 1 & \\
\hline \multicolumn{4}{|l|}{ APLOUSOBRANCHIA } \\
\hline \multicolumn{4}{|l|}{ POLYCITORIDAE } \\
\hline Eudistoma clavatum Rocha \& Bonnet, 2008 & $\mathrm{~N}$ & 1 & \\
\hline \multicolumn{4}{|l|}{ POLYCLINIDAE } \\
\hline Aplidium accarense (Millar, 1953) & $\mathrm{C}$ & 1 & \\
\hline Aplidium sp. & & & 1 \\
\hline \multicolumn{4}{|l|}{ DIDEMNIDAE } \\
\hline Didemnum cineraceum (Sluiter, 1898) & $\mathrm{C}$ & & 1 \\
\hline Didemnum granulatum Tokioka, 1954 & $\mathrm{C}$ & 2 & 1 \\
\hline Didemnum rodriguesi Rocha \& Monniot, 1993 & $\mathrm{C}$ & & 1 \\
\hline Didemnит sp. A & & & 2 \\
\hline Didemnum sp. B & & & 1 \\
\hline Didemnum sp. C & & 1 & 1 \\
\hline Didemnum sp. D & & & 1 \\
\hline Didemnum sp. E & & 1 & \\
\hline Didemnum sp. F & & & 1 \\
\hline Didemnum sp. G & & 1 & \\
\hline Trididemnum orbiculatum (Van Name, 1902) & $\mathrm{N}$ & 1 & \\
\hline Diplosoma listerianum (Milne-Edwards, 1841) & $\mathrm{C}$ & 1 & \\
\hline Lissoclinum fragile (Van Name, 1902) & $\mathrm{C}$ & & 1 \\
\hline Lissoclinum perforatum (Giard, 1878) & $\mathrm{C}$ & 1 & 2 \\
\hline Polysyncraton amethysteum Van Name, 1902 & $\mathrm{~N}$ & & 1 \\
\hline \multicolumn{4}{|l|}{ STOLIDOBRANCHIA } \\
\hline \multicolumn{4}{|l|}{ STYELIDAE } \\
\hline Styela canopus (Savigny, 1816) & $\mathrm{C}$ & & 4 \\
\hline Botrylloides nigrum Herdman, 1886 & $\mathrm{~N}$ & 2 & \\
\hline Botryllus planus (Van Name, 1902) & $\mathrm{C}$ & & 3 \\
\hline \multicolumn{4}{|l|}{ PYURIDAE } \\
\hline Pyura vittata (Stimpson, 1852) & $\mathrm{C}$ & 1 & 6 \\
\hline
\end{tabular}


Pyuridae, Styelidae e Cionidae. As espécies coloniais encontradas estão divididas entre as seguintes famílias: Perophoridae, Polycitoridae, Polyclinidae, Didemnidae e Styelidae. Dentre os didemnídeos, há sete amostras que não puderam ser identificadas em nível de espécie: cinco delas devido à ausência de larvas e as outras duas por não terem sido encontradas espécies semelhantes descritas na literatura (espécies novas prováveis). Apenas cinco espécies podem ser consideradas como nativas para o Atlântico; quatro são também atlânticas, mas criptogênicas na região devido à distribuição disjunta; cinco são classificadas como criptogênicas de ampla distribuição mundial; e uma pode ser considerada como um caso certo de introdução, Ciona intestinalis (Linnaeus, 1767).

Para a costa brasileira, a distribuição dessas espécies nos estados pode ser conferida na tabela II.

As espécies apresentadas neste trabalho são, em grande parte, coincidentes com registros anteriores para o litoral de São Paulo (VAN NAME, 1945; MillaR, 1958; Rodrigues \& Rocha, 1993; Rocha \& MonNiot, 1993; RodRIGUEs et al., 1998), sendo que os únicos registros novos para a região são Eudistoma clavatum Rocha \& Bonnet, 2008, Aplidium sp., Lissoclinum perforatum (Giard, 1878) e Botryllus planus (Van Name, 1902). Entretanto, o fato das outras espécies já terem sido registradas anteriormente não significa que sejam necessariamente nativas do sudeste brasileiro.

Entre as espécies nativas encontradas estão Trididemnum orbiculatum (Van Name, 1902), Polysyncraton amethysteum (Van Name, 1902) e Perophora viridis Verril, 1871. As três espécies apresentam a maioria dos registros no oceano Atlântico oeste (Figs. 1-3). A ausência de T. orbiculatum entre a Bahia e o Ceará (Tab. II) pode indicar apenas a falta de coletas na região, já que a espécie costuma formar colônias pequenas e discretas e seria registrada apenas em levantamentos mais intensivos. Polysyncraton amethysteum (Van Name, 1902) está presente ao longo de toda a costa brasileira, mas existe dúvida se esta espécie seria a mesma daquela presente no Caribe, pois as larvas no material do Caribe são bem maiores (T. M. Lotufo, com. pess.) e VAN NAME (1945) reporta que as colônias estudadas por ele (Bermudas, Flórida e Caribe) são pequenas (com $3 \mathrm{~cm}$ de diâmetro no máximo) e uma cloaca central, enquanto as colônias brasileiras atingem muitos centímetros de diâmetro. Diferenças também são relatadas por MiLlaR $(1953,1977)$ para material coletado respectivamente em Gana e Pernambuco, como por exemplo, larvas com 8 pares de ampolas ectodérmicas, enquanto larvas coletadas em outros pontos da costa brasileira apresentam apenas 6 pares de ampolas (RMR,
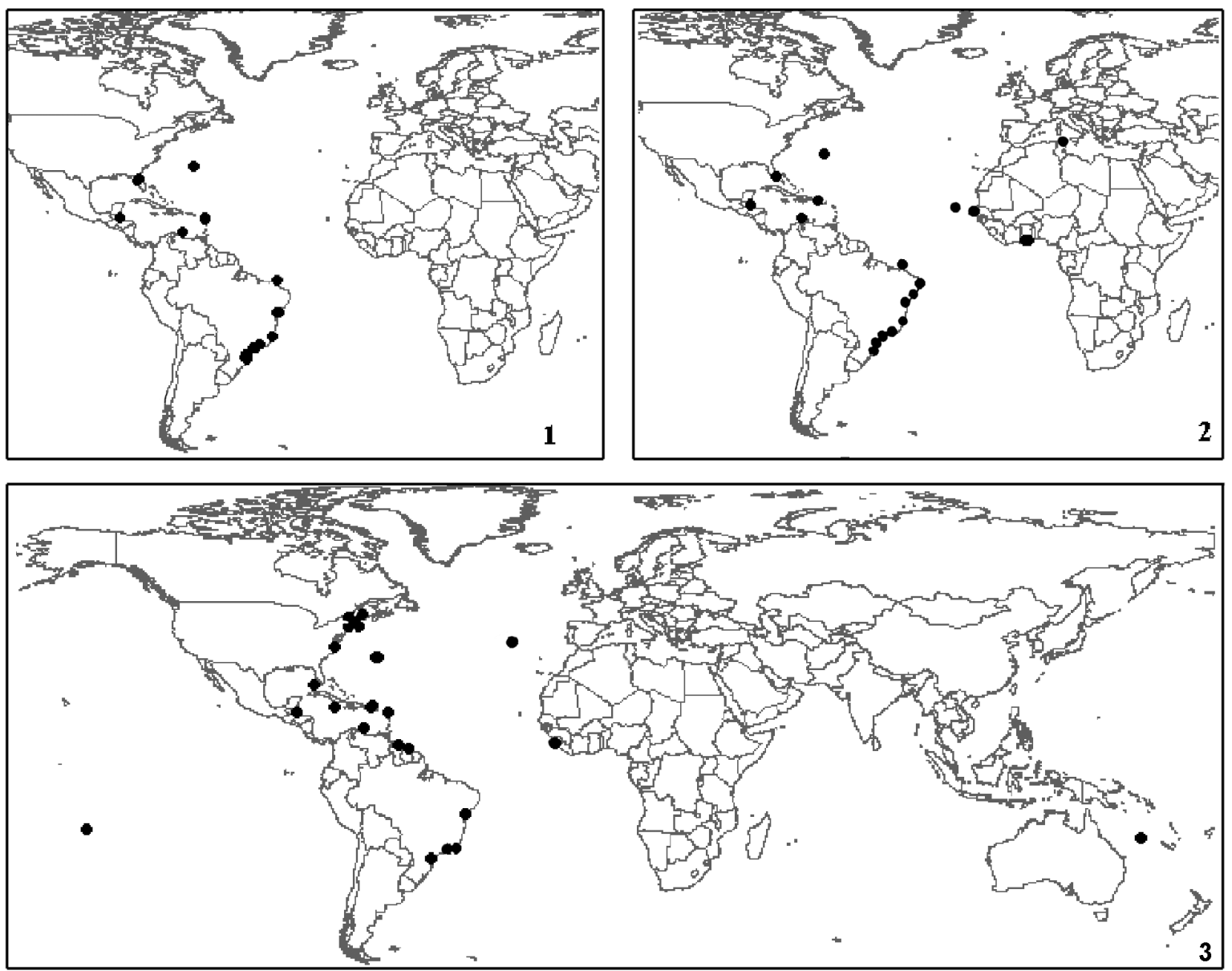

Figs 1-3. Distribuição geográfica de: 1, Trididemnum orbiculatum (Van Name, 1902); 2, Polysyncraton amethysteum (Van Name, 1902); 3, Perophora viridis (Verril, 1871). 
observação pessoal). Há necessidade, pois, de uma revisão da espécie. Os registros africanos podem indicar que a distribuição original da espécie é mais ampla incluindo toda a região tropical do Atlântico, mas os registros na Tunísia (PERES, 1954) podem indicar introduções. Os registros de Perophora viridis na Africa são atribuídos à introdução por MonNIOT (1974).

Botryllus planus também ocorre apenas no Atlântico oeste, mas com distribuição disjunta entre o Caribe e a costa brasileira (Fig. 4), caracterizando uma espécie criptogênica. Entretanto, todos os locais de registro no Brasil são fortemente influenciados por portos, indicando alta probabilidade de que a espécie tenha sido introduzida na costa brasileira.

Aplidium accarense (Millar, 1953) apresenta registros nos dois lados do Atlântico (Fig. 5). Entretanto, por estar restrito a poucas localidades das regiões sudeste e sul do Brasil (Tab. II) e ao noroeste da África (localidades distantes no momento de separação destes continentes) não se pode atribuir a distribuição desta espécie à vicariância. Como não se conhece a área de origem da mesma, é classificada como criptogênica devido à distribuição disjunta. Em Santa Catarina já foi encontrada em ambiente natural (RocHA et al., 2005), mas é muito comum sobre cultivos de mexilhões (RocHA et al., 2009). Com distribuição nos dois lados do Atlântico, encontra-se também Lissoclinum perforatum, mas com registros na Europa e não na África (Fig. 6), caracterizando mais uma vez uma espécie criptogênica. Entretanto, é provável que a distribuição original compreenda apenas o Atlântico leste e Mediterrâneo, onde a espécie é comum. Isto porque em Guadalupe (América Central) foi encontrada uma única colônia (MonNiot, 1983), sendo também bastante rara nos locais de registro brasileiros (RochA et al., 2005).

Didemnum cineraceum (Sluiter, 1898) apresenta poucos registros no mundo. $\mathrm{Na}$ costa brasileira foi encontrada unicamente em São Paulo, no canal de São Sebastião (RodriguEs et al., 1998) e agora em Alcatrazes (Fig. 7). Forma colônias grandes e conspícuas, de fácil identificação devido à larva muito grande e gemípara e, portanto, já teria sido encontrada se estivesse presente em outras regiões bem estudadas como Rio de Janeiro, Paraná e Santa Catarina. Neste contexto, apesar de classificada como criptogênica, existem fortes indícios de que se trata de uma introdução.

Nos três casos, A. accarense, $L$. perforatum e $D$. cineraceum não apresentam registro anterior a 1998

Tabela II. Distribuição das espécies de ascídias registradas em duas localidades do Arquipélago de Alcatrazes, São Paulo, na costa brasileira. 1, Herdman (1886); 2, Michaelsen (1923); 3, Van Name (1945); 4, Moure et al. (1954); 5, Buornberg (1956); 6, Millar (1958); 7, Rodrigues (1962); 8, Rodrigues (1966), 9. Monniot (1969/70); 10, Costa (1969); 11, Millar (1977); 12, Rocha \& Monniot (1993); 13, Rodrigues \& Rocha (1993); 14, Rocha \& Monniot (1995); 15, Rocha \& NAsser (1998); 16, Rodrigues et al. (1998); 17, Rocha \& MoReno (2000); 18, T. Lotufo, com. pess. (2002); 19, Rocha \& Costa (2005); 20, Rocha et al. (2005); 21, Rocha \& Faria (2005); 22, Rocha \& Kremer (2005); 23, Lotufo \& Silva (2005); 24, Rocha \& Bonnet (2008).

\begin{tabular}{|c|c|c|c|c|c|c|c|c|c|c|c|c|}
\hline \multirow[t]{2}{*}{ Espécies } & \multicolumn{11}{|c|}{ Estados Brasileiros } & \multirow[t]{2}{*}{ Referências } \\
\hline & $\mathrm{SC}$ & PR & SP & RJ & $\mathrm{ES}$ & $\mathrm{BA}$ & $\mathrm{AL}$ & $\mathrm{PE}$ & $\mathrm{PB}$ & RN & $\mathrm{CE}$ & \\
\hline $\begin{array}{l}\text { Ciona intestinalis } \\
\text { (Linnaeus, 1767) }\end{array}$ & & & $\mathrm{X}$ & $\mathrm{X}$ & & & & & & & & $2,6,10,16,18$ \\
\hline $\begin{array}{l}\text { Perophora viridis } \\
\text { Verril, } 1871\end{array}$ & $\mathrm{X}$ & & $\mathrm{X}$ & $\mathrm{X}$ & & $X$ & & & & & & $10,16,17,18$ \\
\hline $\begin{array}{l}\text { Eudistoma clavatum } \\
\text { Rocha \& Bonnet, } 2008\end{array}$ & $\mathrm{X}$ & & $\mathrm{X}$ & & & & & & & & & 24 \\
\hline $\begin{array}{l}\text { Aplidium accarense } \\
\text { (Millar, 1953) }\end{array}$ & $\mathrm{X}$ & & $\mathrm{X}$ & & & & & & & & & 16,20 \\
\hline $\begin{array}{l}\text { Didemnum cineraceum } \\
\text { (Sluiter, 1898) }\end{array}$ & & & $\mathrm{X}$ & & & & & & & & & 16 \\
\hline $\begin{array}{l}\text { Didemnum granulatum } \\
\text { Tokioka, } 1954\end{array}$ & $\mathrm{X}$ & $\mathrm{X}$ & $\mathrm{X}$ & & & $\mathrm{X}$ & & & $\mathrm{X}$ & $\mathrm{X}$ & $\mathrm{X}$ & $\begin{array}{l}14,15,16,18 \\
20,21,22,23\end{array}$ \\
\hline $\begin{array}{l}\text { Didemnum rodriguesi } \\
\text { Rocha \& Monniot, } 1993\end{array}$ & $\mathrm{X}$ & $\mathrm{X}$ & $\mathrm{X}$ & $\mathrm{X}$ & & & & & & & & $12,15,16,18,20,21$ \\
\hline $\begin{array}{l}\text { Trididemnum orbiculatum } \\
\text { (Van Name, 1902) }\end{array}$ & $\mathrm{X}$ & $\mathrm{X}$ & $\mathrm{X}$ & $\mathrm{X}$ & $\mathrm{X}$ & $\mathrm{X}$ & & & & & $\mathrm{X}$ & $13,16,17,18,20,21$ \\
\hline $\begin{array}{l}\text { Diplosoma listerianum } \\
\text { (Milne-Edwards, 1841) }\end{array}$ & $\mathrm{X}$ & $\mathrm{X}$ & $\mathrm{X}$ & $\mathrm{X}$ & $\mathrm{X}$ & $\mathrm{X}$ & $\mathrm{X}$ & & & & & $\begin{array}{l}1,3,13,15,16 \\
18,20,21,22\end{array}$ \\
\hline $\begin{array}{l}\text { Lissoclinum fragile } \\
\text { (Van Name, 1902) }\end{array}$ & $\mathrm{X}$ & $\mathrm{X}$ & $\mathrm{X}$ & $\mathrm{X}$ & & & & $\mathrm{X}$ & & & $\mathrm{X}$ & $16,18,20,22$ \\
\hline $\begin{array}{l}\text { Lissoclinum perforatum } \\
\text { (Giard, 1878) }\end{array}$ & $\mathrm{X}$ & & $\mathrm{X}$ & $\mathrm{X}$ & & & & & & $\mathrm{X}$ & & $16,18,20$ \\
\hline $\begin{array}{l}\text { Polysyncraton amethysteum } \\
\text { Van Name, } 1902\end{array}$ & $\mathrm{X}$ & $\mathrm{X}$ & $\mathrm{X}$ & $\mathrm{X}$ & $\mathrm{X}$ & $\mathrm{X}$ & & $\mathrm{X}$ & & $\mathrm{X}$ & $\mathrm{X}$ & $\begin{array}{l}4,5,6,7,11,12,13 \\
16,17,20,21,23\end{array}$ \\
\hline $\begin{array}{l}\text { Botrylloides nigrum } \\
\text { Herdman, } 1886\end{array}$ & $\mathrm{X}$ & $\mathrm{X}$ & $\mathrm{X}$ & $\mathrm{X}$ & $\mathrm{X}$ & $\mathrm{X}$ & $\mathrm{X}$ & $\mathrm{X}$ & & & & $\begin{array}{l}7,13,16,18,19 \\
20,22\end{array}$ \\
\hline $\begin{array}{l}\text { Botryllus planus } \\
\text { (Van Name, 1902) }\end{array}$ & & $\mathrm{X}$ & $\mathrm{X}$ & $\mathrm{X}$ & & $\mathrm{X}$ & & & & & $\mathrm{X}$ & $10,18,22,23$ \\
\hline $\begin{array}{l}\text { Styela canopus } \\
\text { (Savigny, 1816) }\end{array}$ & $\mathrm{X}$ & $\mathrm{X}$ & $\mathrm{X}$ & $\mathrm{X}$ & $\mathrm{X}$ & $\mathrm{X}$ & & $\mathrm{X}$ & & $\mathrm{X}$ & & $9,16,18,20,22$ \\
\hline $\begin{array}{l}\text { Pyura vittata } \\
\text { (Stimpson, 1852) }\end{array}$ & & & $\mathrm{X}$ & & & $\mathrm{X}$ & & $X$ & & & & $8,11,16,18$ \\
\hline
\end{tabular}


(RoDRIGUES et al., 1998), apesar da região de São Sebastião em São Paulo ter sido razoavelmente bem estudada desde a década de 60 (mais um forte indício de que estas espécies tenham sido introduzidas recentemente na região).

Didemnum rodriguesi Rocha \& Monniot, 1993 também é uma espécie de distribuição disjunta, sendo encontrada no Brasil, na Nova Caledônia (Oceania) e na África do Sul (Fig. 8). Como a maior parte dos registros ocorreu no Brasil, apesar das outras duas áreas serem bem estudadas, considerou-se $D$. rodriguesi uma espécie nativa neste país, com introduções nas outras duas localidades.

Classificadas como criptogênicas com ampla distribuição mundial, estão Didemnum granulatum Tokioka, 1954 (Fig. 9); Diplosoma listerianum (MilneEdwards, 1841) (Fig. 10); Lissoclinum fragile (Van Name, 1902) (Fig. 11); Styela canopus (Savigny, 1816) (Fig. 12) e Pyura vittata (Stimpson, 1852) (Fig. 13). No caso de Botrylloides nigrum Herdman, 1886 (Fig. 14), o fato do maior número de registros localizarem-se no oceano Atlântico Oeste caracteriza esta espécie como nativa nesta região e levanta a hipótese de introdução nas outras localidades.

Ciona intestinalis é a única espécie encontrada que se trata de um caso certo de introdução no Brasil, pois tem como distribuição original o noroeste da Europa (LAMBERT, 2001) e apresenta atualmente ampla distribuição mundial (Fig. 15). Esta espécie foi coletada no Rio de
Janeiro (Costa, 1969) e São Paulo (Millar, 1958) no final da década de 1950 e apenas no Rio de Janeiro se manteve como uma espécie relativamente comum durante 20 anos, tendo desaparecido no início da década de 1980 (A. Junqueira, com. pess.). Na coleção de Ascidiacea do Departamento de Zoologia da UFPR existe um lote de animais coletados em 1957 no litoral do Paraná (registro não publicado), mas a espécie não foi mais encontrada na região. Em São Paulo, o registro de RodRigues et al. (1998) refere-se a um único exemplar, assim como no presente trabalho, o que indica que a espécie não conseguiu se estabelecer no local, mas está presente e pode vir a se tornar um problema se não for controlada. No estuário de São Francisco, EUA (onde a espécie é invasora e ocorre em altas densidades) $C$. intestinalis reduz a riqueza de espécies e altera a composição de espécies da comunidade (BLum et al., 2007).

Se por um lado foram encontradas algumas espécies novas (Aplidium sp., Didemnum sp. A, Didemnum sp. G) no Arquipélago de Alcatrazes, por outro é preocupante a presença de uma espécie introduzida (Ciona intestinalis) e pelo menos quatro criptogênicas com grande chance de serem também introduzidas na região. Deve-se considerar ainda que apenas duas localidades dentro do Arquipélago foram estudadas em uma única campanha de coletas e que estas localidades apresentam poucas espécies em comum (Tab. I), o que indica que a riqueza de espécies
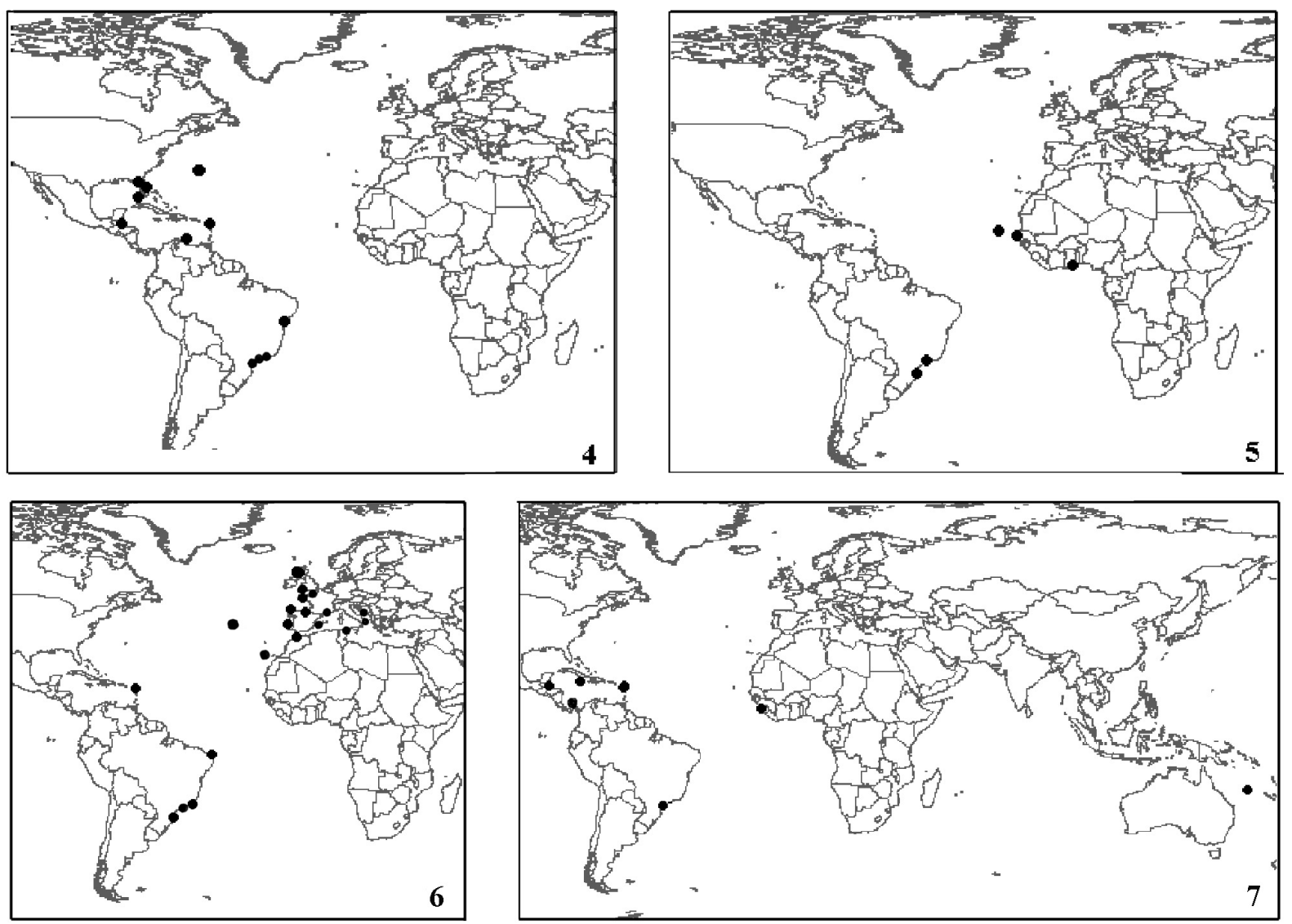

Figs. 4-7. Distribuição geográfica de: 4, Botryllus planus (Van Name, 1902); 5, Aplidium accarense (Millar, 1953); 6, Lissoclinum perforatum (Giard, 1878); 7, Didemnum cineraceum (Sluiter, 1898). 

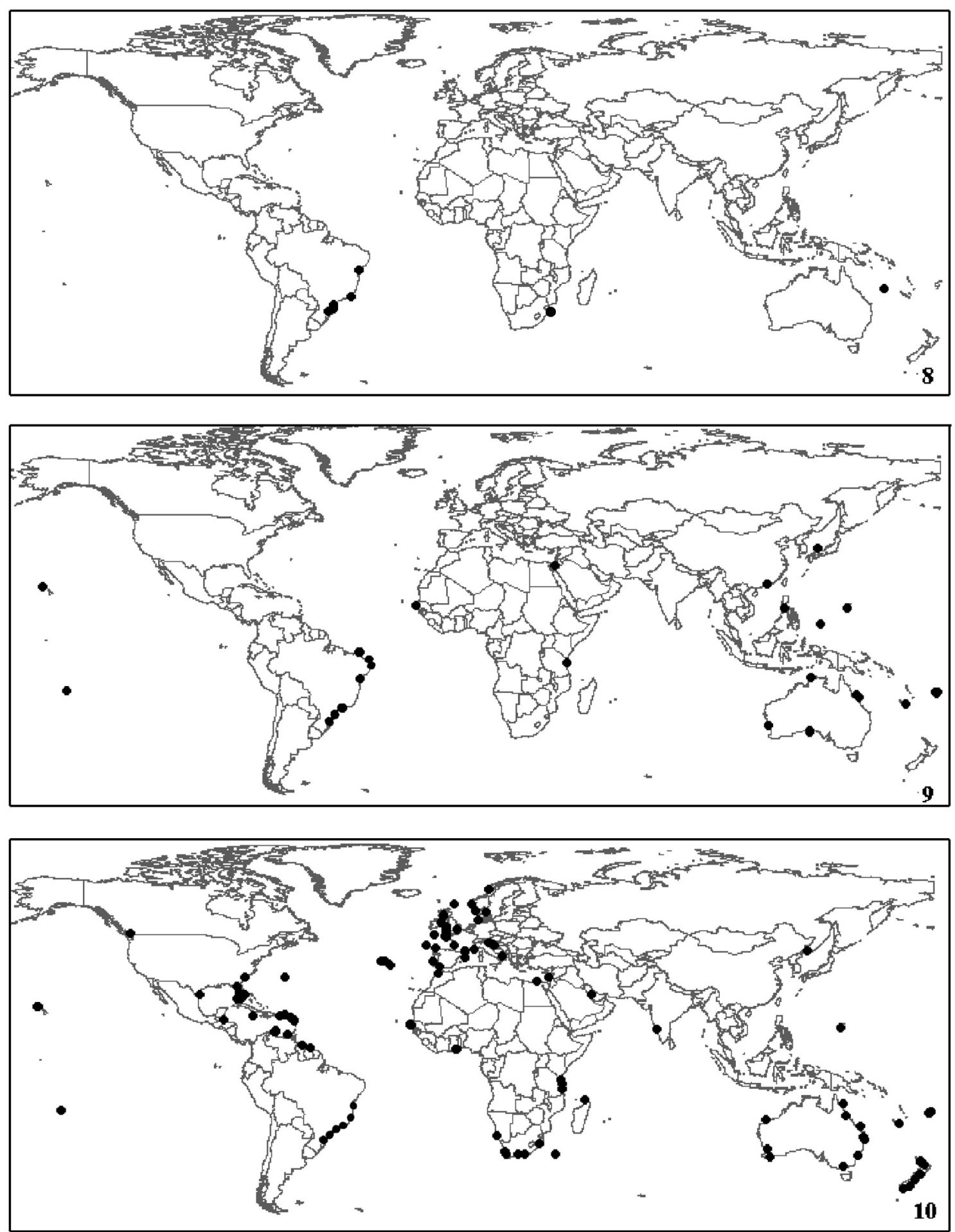

Figs. 8-10. Distribuição geográfica de: 8, Didemnum rodriguesi (Rocha \& Monniot, 1993); 9, Didemnum granulatum Tokioka, 1954; 10, Diplosoma listerianum (Milne-Edwards, 1841)

do Arquipélago pode ser bem maior. Nenhuma das espécies apresentou uma grande abundância característica de espécies invasoras, mas sabe-se que pode haver um longo período de estabelecimento e que qualquer modificação ambiental pode iniciar um processo de aumento descontrolado da população de uma espécie, o que poderia colocar em risco a fauna local (STACHowicz et al., 2002). Embarcações de recreio constituem os principais dispersores locais de espécies introduzidas que chegam aos grandes portos com movimento de navios internacionais (WASSON et al., 2001). Como o Arquipélago de Alcatrazes é controlado pela Marinha do Brasil e a circulação de embarcações é bastante restrita, a chegada destas espécies introduzidas 

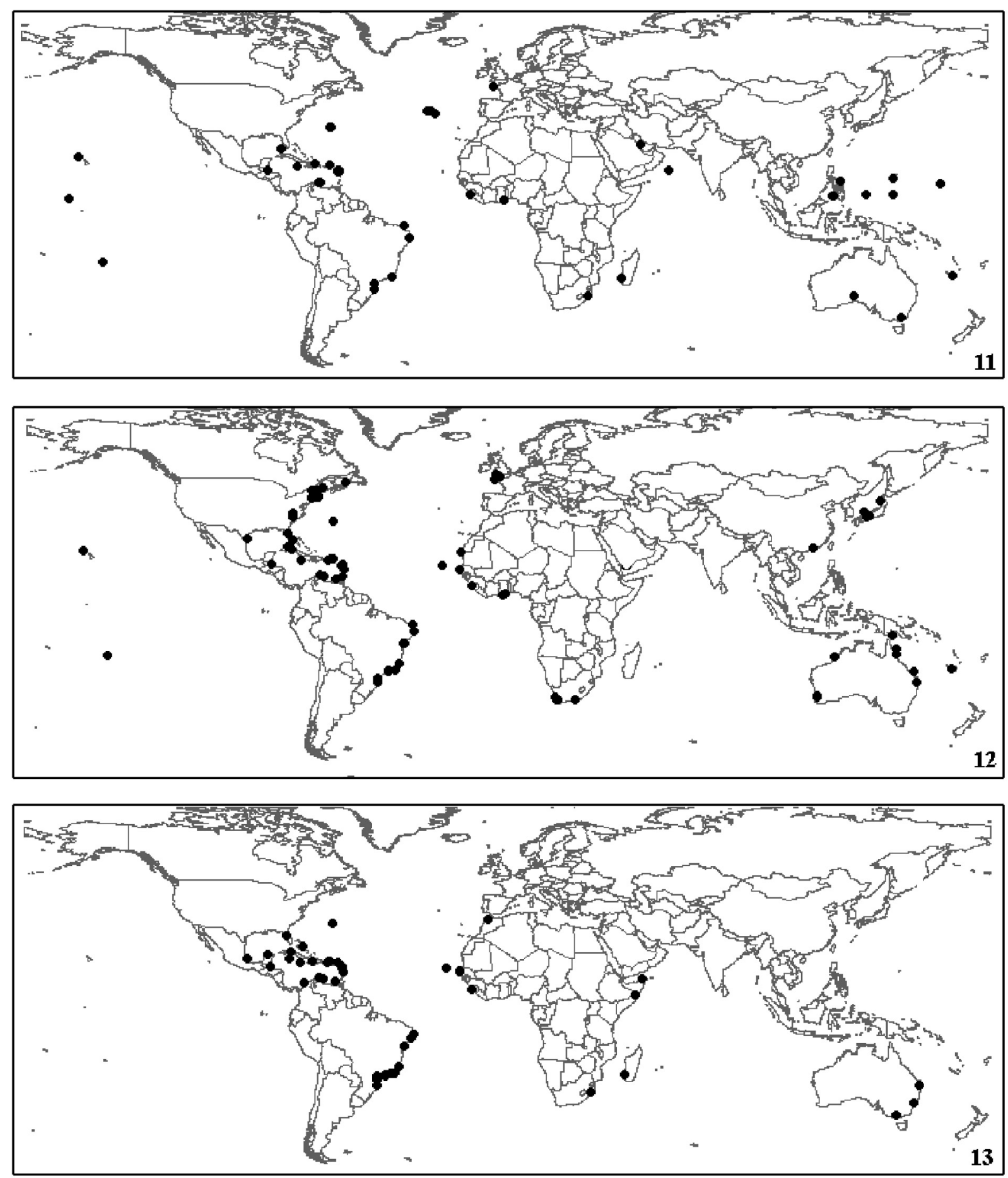

Figs. 11-13. Distribuição geográfica de: 11, Lissoclinum fragile (Van Name, 1902); 12, Styela canopus (Savigny, 1816); 13, Pyura vittata (Stimpson, 1852).

indica que as mesmas devem estar se dispersando por meio de larvas capazes de viajar pelo menos os $36 \mathrm{~km}$ que separam as ilhas do continente, sugerindo que a fauna da região continental adjacente também esteja ameaçada. Assim como as ascídias, outros grupos taxonômicos devem ser inventariados para uma maior compreensão do processo de bioinvasão na região. O informe sobre espécies exóticas invasoras que afetam o ambiente marinho (MMA, 2006) indicou a presença de 66 espécies na costa brasileira, sendo 10 macroalgas, 38 zoobentos e 4 peixes, grupos que poderiam afetar diretamente o arquipélago. Portanto, o manejo desta unidade de conservação deve considerar a presença do porto de São Sebastião como fonte de estresse e o monitoramento constante das espécies introduzidas para detecção precoce e erradicação. 

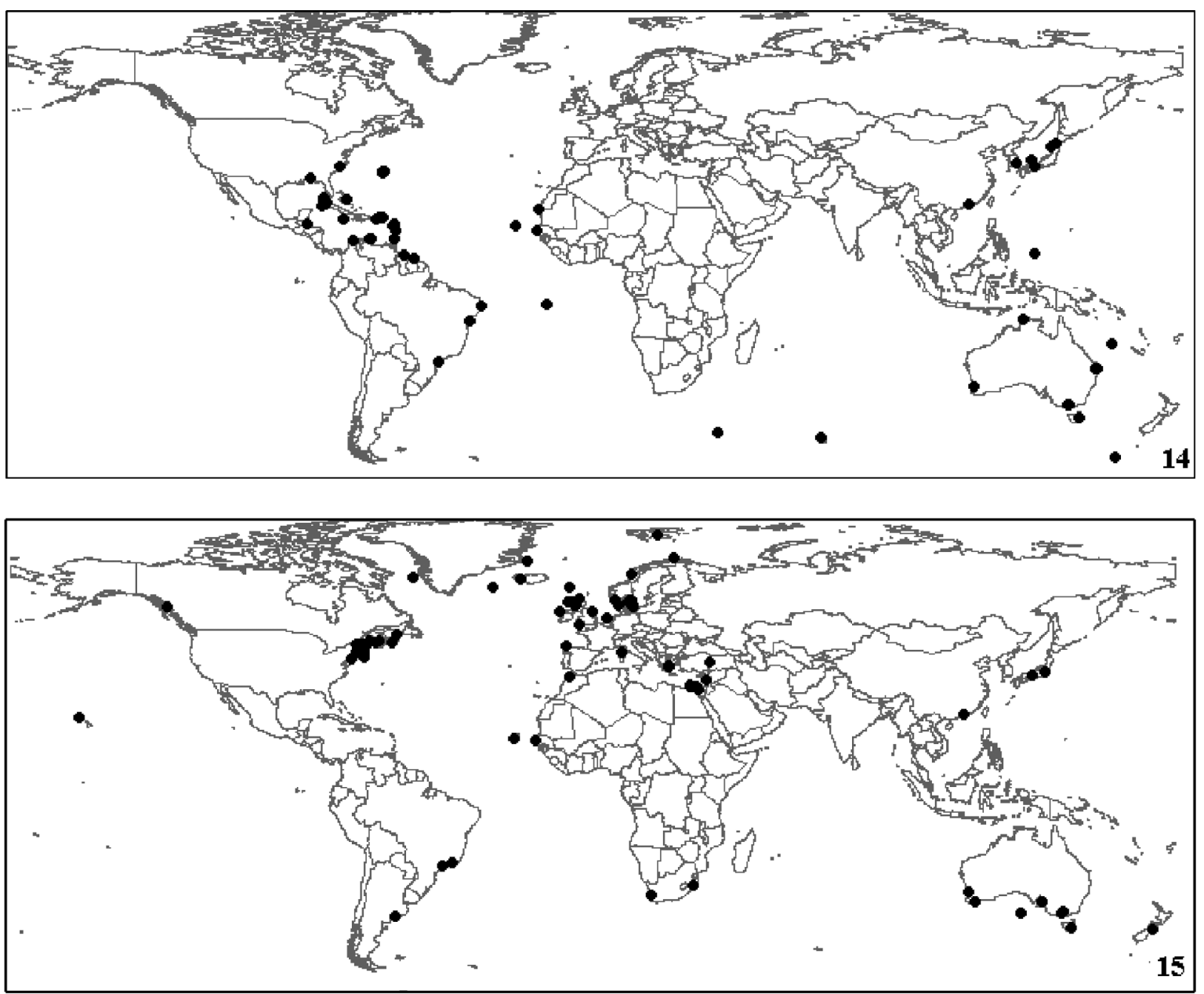

Figs. 14-15. Distribuição geográfica de: 14, Botrylloides nigrum Herdman, 1886; 15, Ciona intestinalis (Linnaeus, 1767).

Agradecimentos. Ao CNPq pela concessão das bolsas, de pesquisa do autor sênior e PIBIC de N. Y. K. Bonnet. Ao Dr. Roberto G. S. Berlinck e a FAPESP pelo apoio financeiro (processo $\left.\mathrm{n}^{\circ} 05 / 60175-2\right)$ que possibilitou a realização das coletas. Ao Dr. James Roper e Dr. Rafael Metri pelo auxílio no trabalho de campo. Ao Centro de Microscopia Eletrônica da Universidade Federal do Paraná que possibilitou o estudo de espículas dos Didemnídeos. Contribuição ${ }^{\circ} 1718$ do Departamento de Zoologia da Universidade Federal do Paraná

\section{REFERÊNCIAS BIBLIOGRÁFICAS}

BJoRnBerg, T. K S. 1956. Ascídias da costa sul do Brasil (nota prévia). Ciência e Cultura 8(3):164-65.

Blum, J. C.; Chang, A. L.; Liljesthrom, M.; Schnk, M. E.; Stinberg, M. K. \& Ruiz, G. M. 2007. The non-native solictary ascidian Ciona intestinalis (L.) depresses species richness. Journal of Experimental Marine Biology and Ecology 342:5-14.

CARlton, J. T. 1996. Biological invasions and cryptogenic species. Ecology 77(6):1653-1655.

Carlton, J. T. \& Geller J. B. 1993. Ecological roulette: the global transport of nonindigenous marine organisms. Science 261:78-82

Cohen, A. N. \& Carlton, J. T. 1998. Accelerating invasion rate in a highly invaded estuary. Science 279:555-558.

Costa, H. R. 1969. Notas sobre os Ascidiacea brasileiros. IV. Ordem Phlebobranchia (Lahille, 1887). Atas da Sociedade de Biologia do Rio de Janeiro 12(5-6):289-292.

Davis, M. H. \& Davis, M. E. 2005. Styela clava (Tunicata: Ascidiacea): a new addition to the fauna of the Portuguese coast. Journal of the Marine Biological Association of the United Kingdom 85:403-404.

Dias, G. M. \& Rodrigues, S. A. 2004. Didemnum tetrahedrum sp. nov., a new Didemnum (Tunicata: Ascidiacea) species from south-eastern Brazil. Journal of the Marine Biological Association of the United Kingdom 84:1227-1228.

Herdman, W. A. 1886. Report on the Tunicata collected during the Voyage of H.M.S. Challenger during the years 18731876. Part 2, Ascidiae compositae. Edinburg, Report of the Scientific Results of the Voyage of H.M.S. Challenger during the years $1873-76$, v.14. $429 \mathrm{p}$

LAMBERT, G. 2001. A global overview of ascidians introductions and their possible impact on the endemic fauna. In: SAWADA, H.; Yokosawa, H. \& Lambert, C. C. eds. The Biology of Ascidians, Tokyo, Springer Verlag. p.249-257.

2004. The south temperate and Antarctic ascidian Corella eumyota reported in two harbours in north-western France. Journal of the Marine Biological Association of the United Kingdom 84:239-241.

2007. The nonindigenous ascidian Molgula ficus in Califórnia. Cahiers de Biologie Marine 48:95-102.

Lotufo, T. M. C. \& Silva, A. M. B. 2005. Ascidiacea. In: CAscon, H. M. \& Lotufo, T. M. C. eds. Biota Marinha da Costa Oeste do Ceará. Fortaleza, Ministério do Meio Ambiente. p.222247.

Michaelsen, W. 1923. Neue und altbekannte Ascidien aus dem Re ichsmuseum zu Stockholm. Mitteilungen aus dem Zoologischen Museum 40:1-60.

MillaR, R. H. 1953. On the collection of ascidians from the Gold Coast. Proceedings of the Zoological Society of London $123(2): 277-325$ 
1958. Some ascidians from Brazil. Annals and Magazine of Natural History 13(1):497-514.

1977. Ascidians (Tunicata: Ascidiacea) from the Northern and North-eastern brazilian shelf. Journal of Natural History 11(2): 169-223.

Minchin, D.; Davis, M. H. \& Davis, M. E. 2006. Spread of the Asian tunicate Styela clava Herdman, 1882 to the east and southwest coasts of Ireland. Aquatic Invasions 1(2):91-96.

MMA (Ministério do Meio Ambiente). 2006. Espécies Exóticas Invasoras: Situação Brasileira. Brasília, Ministério do Meio Ambiente, Secretaria de Biodiversidade e Florestas. 23p.

Monniot, C. 1969-1970. Ascidies phlebobranches et stoldidobranches. In: Campagne de ia Calypso au large des cotes de l'Amerique du Sud. Annales de l'Institut Oceanographique 47:33-59.

1974. Ascidies littorales et Bathyales récoltées au cours de la campagne Biaçores: Phlébobranches et Stolidobranches. Bulletin du Muséum National d'Histoire Naturelle 3e ser 173(251):1327-1352.

Monniot, F. 1983. Ascidies littorales de Guadaloupe. I. Didemnidae. Bulletin du Muséum National d'Histoire Naturelle 4e ser 5(1):5-49.

Moure, J. S.; Buornberg, T. K. S. \& Loureiro, T. St. 1954. Protochordata ocorrentes na entrada da Baí de Paranaguá. Dusenia 5(5-6):233-242.

Peres, J. M. 1954. Contribution a l'étude des ascidies de Tunisie. Bulletin de la Station Océanographique de Salammbo 49:3-21.

Projeto Alcatrazes. 2008. Projeto Alcatrazes, pela criação do Parque Nacional. Disponível em: <http:// www.alcatrazes.org.br/home>. Acesso em: 20.06.2008.

Rосна, R. M. 2002. Bostricobranchus digonas Abbott (Ascidiacea, Molgulidae) in Paranaguá Bay, Paraná, Brazil. A case of recent invasion? Revista Brasileira de Zoologia 19:157-161.

Rocha, R. M. \& Bonnet, N. Y. K. 2008. Eudistoma clavatum sp. nov. (Tunicata: Ascidiacea: Polycitoridae). JMBA2 Biodiversity Records 5925:1-5. Disponível em: <http:// www.mba.ac.uk/jmba/jmba2biodiversityrecords.php>. Acesso em: 20.06.2008.

Rocha, R. M. \& Costa, L. V. G. 2005. Ascidians (Urochordata: Ascidiacea) from Arraial do Cabo, RJ, Brazil. Iheringia, Série Zoologia, 95(1):57-64

Rocha, R. M. \& Faria, S. B. 2005. Ascidians at Currais Islands, Paraná, Brazil: taxonomy and distribution. Biota Neotropica 5(2). Disponível em: <http://www.biotaneotropica.org.br/ $\mathrm{v} 5 \mathrm{n} 2 / \mathrm{pt} / \mathrm{item}$ ?article>. Acesso em: 20.06.2008.

Rocha, R. M. \& Kremer, L. P. 2005. Introduced ascidians in Paranaguá Bay, Paraná, southern Brazil. Revista Brasileira de Zoologia 22(4):1170-1184.

Rocha, R. M.; Kremer, L. P.; Baptista, M. S. \& Metri, R. 2009. Bivalve cultures provide habitat for exotic tunicates in southern Brazil. Aquatic Invasions 4:195-205.

Rocha, R. M. \& Monniot, F. 1993. Didemnum rodriguesi sp. nov., a new didemnid tunicate common to southern Brazil and
New Caledonia. Annales del'Institut Océanographique 69(2):261-265.

1995. Taxonomic and ecological notes on some Didemnum species (Ascidiacea, Didemnidae) for São Sebastião Channel, south-east Brazil. Revista Brasileira de Biologia 55(4):639649.

Rocha, R. M. \& Moreno, T. R. 2000. Ascidians associated with Eudistoma carolinense Van Name, 1945. With description of a new species of Polycarpa. Ophelia 52(1):9-16.

Rocha, R. M.; Moreno, T. R. \& Metri, R. 2005. Ascídias (Tunicata, Ascidiacea) da Reserva Biológica Marinha do Arvoredo, Santa Catarina, Brasil. Revista Brasileira de Zoologia 22(2):461-476.

Rocha, R. M. \& NAsSER, C. M. 1998. Some ascidians (Tunicata, Ascidiacea) from Parana State, Southern Brasil. Revista Brasileira de Zoologia 15(3):633-642.

Rodrigues, S. A. 1962. Algumas ascídias do litoral sul do Brasil. Boletim da Faculdade de Filosofia, Ciências e Letras Universidade de São Paulo, Série Zoologia, 24:193-216. 1966. Notes on Brazilian ascidians. I. Papéis Avulsos do Departamento de Zoologia 19(8):95-115.

1977. Notes on Brazilian ascidians. II. On the records of Polyandrocarpa anguinea (Sluiter) e Polyandrocarpa maxima (Sluiter). Revista Brasileira de Biologia 37(4):721-26.

Rodrigues, S. A. \& Rocha, R. M. 1993. Littoral compound ascidians (Tunicata) from São Sebastião, Estado de São Paulo, Brazil. Proceedings of the Biological Society of Washington 106(4):728-739.

Rodrigues, S. A.; Rocha, R. M. \& Lotufo, T. M. C. 1998. Guia ilustrado para identificação das ascídias do Estado de São Paulo. São Paulo, FAPESP, 190p.

Ruiz, G. M.; Fofonoff, P. W.; Carlton, J. T.; Wonham, M. J. \& Hines, A. H. 2000. Invasion of coastal marine communities in North America: Apparent Patterns, Processes, and Biases. Annual Review of Ecology and Systematics 31:481-531.

Secretaria de Estado dos Transportes. 2008. Governo do Estado de São Paulo, Secretaria dos Transportes. Disponível em: <http://www.transportes.sp.gov.br/v20/portosaosebastiao. asp>. Acesso em: 20.06.2008.

Stachowicz, J.; Terwin, J. R.; Whitlatch, R. B. \& Osman, R. W. 2002. Linking climate change and biological invasions: Ocean warming facilitates nonindigenous species invasions. Proceedings of the National Academy of Science 99(24): 15497-15500.

Van Name, W. G. 1945. The North and South American Ascidians. Bulletin of the American Museum of Natural History 84:1-476.

Wasson, K.; Zabin, C. J.; Bedinger, L.; Diaz, M. C. \& Pearse, J. S. 2001. Biological invasions of estuaries without international shipping: the importance of intraregional transport. Biological Conservation 102:143-153.

Zvyagintsev, A. Yu.; Sanamayan, K. E. \& Koryakova, M. D. 2003. The introduction of the ascidian Molgula manhattensis (De Kay, 1843) into Peter the Great Bay (Sea of Japan). Sessile Organisms 20(1):7-10.

Recebido em outubro de 2007. Aceito em julho de 2008. ISSN 0073-4721

Artigo disponível em: www.scielo.br/isz 